\title{
Light ion EDM search in magnetic storage rings
}

\author{
C. J. G. Onderwater
}

Published online: 20 June 2007

(C) Springer Science + Business Media B.V. 2007

\begin{abstract}
Permanent electric dipole moments (EDMs) violate parity and timereversal symmetry. Within the Standard Model (SM), they require CP violation and are many orders of magnitude below present experimental sensitivity. Many extensions of the SM predict much larger EDMs, which are therefore an excellent probe for the existence of 'new physics.' So far only electrically neutral systems were used for sensitive searches of EDMs. Several techniques, based on storing fast particles in a magnetic storage ring, are being developed to probe charged particles for an EDM. With the introduction of these novel experimental methods, high sensitivity for charged systems, in particular light nuclei, is within reach.
\end{abstract}

Keywords Light ion EDM • Electric dipole moments • Standard model • Magnetic storage rings

\section{Introduction}

The study of the discrete symmetries $\mathcal{C}$ (charge conjugation), $\mathcal{P}$ (parity), and $\mathcal{T}$ (time reversal) has been the source of some of the most important progress in the understanding of fundamental interactions during the past 50 years. On theoretical grounds, it is expected that the combined $\mathcal{C P} \mathcal{T}$ symmetry is respected in all interactions and no experimental evidence exists to the contrary. In the electromagnetic interaction all three symmetries are found to be respected individually. Ample experimental data exists for the full breaking of $\mathcal{C}$ and $\mathcal{P}$ in the weak interaction and increasingly more precise data on hadronic $\mathcal{C P}$ breaking becomes available from experiments with $\mathrm{K}, \mathrm{B}$ and D-mesons. The strong interaction seems to conserve

The author represents the Storage Ring EDM Collaboration.

C. J. G. Onderwater $(\bowtie)$

University of Groningen, Groningen, The Netherlands

e-mail: onderwater@kvi.nl 
all symmetries. Theoretically, there is room for $\mathcal{C P}$ violation via the so-called $\bar{\theta}$ term, which is experimentally limited to a seemingly unnatural value of order $10^{-9}$. No undisputed observation for the violation of $\mathcal{T}$ has been reported so far. Many searches are underway worldwide to set more stringent limits on $\mathcal{T}$ violation [1]. This includes searches for non-zero permanent electric dipole moments (EDMs), which in addition to $\mathcal{T}$ also break $\mathcal{P}$.

\section{Electric dipole moments}

Within the Standard Model (SM), particles acquire non-zero EDMs through third or higher order $\mathcal{C P}$ violating radiative corrections. This makes them extremely small $\left(\sim 10^{-(31 \cdots 38)} \mathrm{e} \cdot \mathrm{cm}\right)$ and far below present detection limits [2]. In many proposed extensions of the SM much larger EDMs are predicted because the need for higherorder is often not present [3]. Therefore, the quest for a non-zero EDM is a very clear way to unambiguously establish the presence of physics beyond the Standard Model or to put constrains on speculative new models.

The EDM of composed systems is build up from the EDMs of its constituents, as well as from $\mathcal{C P}$ violating interactions between them. In particular, different nuclei exhibit different sensitivities to, e.g., $\bar{\theta}$ in the SM or $\mathcal{C P}$ violating terms in new models. Especially for small nuclei firm predictions are possible because the structure calculation can be performed with sufficient reliability. For example, in [4], the sensitivity to quark chromo $\operatorname{EDMs} \tilde{d}_{q}$ for the neutron $(n)$ and deuteron $(\mathcal{D})$ are found to be

$$
d_{n}=-0.01 \tilde{d}_{d}+0.49 \tilde{d}_{u}, \quad d_{\mathcal{D}}=-4.67 \tilde{d}_{d}+5.22 \tilde{d}_{u} .
$$

A check as to whether $\bar{\theta}$ is the source of observed EDMs is the relation (Marciano, private communication)

$$
d_{p}: d_{\mathcal{D}}: d{ }_{H e} \simeq 3: 1:-3
$$

The large sensitivity to new physics (see e.g. [5]) and the relative simplicity of calculating the nuclear wavefunction, make it clear that small nuclei hold great discovery potential and should therefore be vigorously pursued.

\section{EDM detection}

The most sensitive experimental signature of an EDM is the dependence of the spin precession frequency on the strength of an externally applied electric field. For a precise measurement, both the electric field felt by the particle and the observation time need to be as large as possible. So far, this has limited searches to neutral systems (neutrons, atoms and molecules), which do not accelerate in the electric field. Unfortunately, rearrangement of the atomic electrons shields the atomic nucleus from an external electric field [6], in particular for small nuclei. Only for large nuclei (e.g. ${ }^{199} \mathrm{Hg}, \mathrm{Ra}$ ) this shielding is incomplete [7].

Direct precise measurement of the EDM of a charged particle is complicated because they quickly accelerate out of the experiment. This can be avoided by storing a fast moving charged particle in a magnetic field. In its rest-frame the particle 
experiences a motional electric field $\vec{E}=\vec{v} \times \vec{B}$. The Lorentz-force keeps the particle from escaping the experiment.

\section{Spin dynamics in electro-magnetic storage rings}

The evolution of the spin orientation (relative to the momentum) for a spin- $1 / 2$ particle in an electromagnetic field $(\vec{E}, \vec{B})$ is described by the so-called Thomas or BMT equation,

$$
\frac{d \vec{S}}{d t}=\frac{e}{m} \vec{S} \times\left[a \vec{B}+\left(a-\frac{1}{\gamma^{2}-1}\right) \vec{\beta} \times \vec{E}+\frac{\eta}{2}(\vec{E}+\vec{\beta} \times \vec{B})\right] \equiv \vec{S} \times \vec{\Omega} .
$$

Here, it was used that $\vec{\mu}=2(1+a)(e / m) \vec{S}$ and $\vec{d}=\eta / 2(e / m) \vec{S}$. Further, it was assumed that $\vec{\beta} \cdot \vec{B}=\vec{\beta} \cdot \vec{E}=0$. In the remainder of this paper, the first two terms between square brackets will be referred to as $\omega_{a}$, whereas the last will be referred to as $\omega_{\eta}$. Several techniques can be devised to gain sensitivity to the EDM, three of which are outlined below.

\subsection{The 'parasitic' way}

It is clear from (3), that the magnetic and electric dipole moments both contribute to the precession $\vec{\Omega}=\vec{\omega}_{a}+\vec{\omega}_{\eta}$. With the assumption that the average electric field in the laboratory system is zero, $\vec{\omega}_{a}=(a e / m) \vec{B}$ and $\vec{\omega}_{\eta}=(\eta e / 2 m)(\vec{\beta} \times \vec{B})$. The magnitude of the precession rate thus depends on the EDM in second order, $\Omega=\sqrt{\omega_{a}^{2}+\omega_{\eta}^{2}} \simeq$ $\omega_{a}\left[1+\frac{1}{2}(\eta \beta / 2 a)^{2}\right]$. More importantly, the precession plane, defined by the vector $\vec{\Omega}$, is tilted with respect to the plane containing the orbits of the particles, defined by $\vec{B}$. The tilt angle is

$$
\phi=\arctan \frac{\omega_{\eta}}{\omega_{a}} \simeq \frac{\eta \beta}{2 a} .
$$

The linear dependence gives a more solid handle on the EDM than the change in frequency and was used to set a limit on the EDM of the muon $d_{\mu}<2.8 \times$ $10^{-19} \mathrm{e} \cdot \mathrm{cm}(95 \%$ C.L.) [8, 9]. The sensitivity of this method is limited, because the vertical polarization component can only grow for a time of the order of the precession period.

\subsection{Frozen spin}

The sensitivity for an EDM is increased if $\omega_{a} \rightarrow 0$, in which case the growth of the vertical spin component continues as long as the beam is polarized. As discussed in [10], this can be accomplished using a radially oriented electric field of strength

$$
E_{r}=\frac{a B c \beta}{1-(1+a) \beta^{2}} \quad \rightarrow \quad \vec{\omega}_{a}=0 .
$$

The EDM manifests itself as a precession of the spin around $\vec{v} \times \vec{B}$, i.e. a growing vertical polarization component parallel to $\vec{B}$. 
The strengths of this method lie in the fact that the growth time of the vertical spin component can be as long as the spin coherence time of the beam and that the electric field in the particle rest frame may be significantly larger than the (break-through limited) one in the laboratory frame, by a factor $\xi=\frac{\beta B_{z}+E_{r}}{E_{r}}=\frac{a+1}{a \gamma^{2}}$. For particles with small $a$ and low $\gamma, \xi \simeq 10-100$.

The statistical precision of this method depends on several factors, such as the initial polarization of the beam $P_{\circ}$, the beam lifetime $\tau$, the polarization coherence time $\tau_{p}$, the effective electric field strength $\xi E_{r}$, the analyzing power of the polarimeter $A$, and the number of detected particles $\epsilon N_{\text {tot }}$. The uncertainty can be described in good approximation by

$$
\sigma_{d} \simeq \frac{4 \hbar}{\sqrt{\tau \tau_{p}} \xi E_{r} P_{\circ} A \sqrt{\epsilon N_{\mathrm{tot}}}}
$$

The front end of the experiment must consist of an intense source of highly polarized particles to maximize $N_{\text {tot }}$ and $P_{\circ}$ and accelerate them to optimize $\xi$. The storage ring must be designed with special care to reach long spin and beam lifetimes $\tau$ and $\tau_{p}$. Optimization of the detection efficiency $\epsilon$ (including the beam lifetime $\tau$ ) and analyzing power $A$ guide the design of the polarimeter.

The dominant source of systematic uncertainty for this technique is an electric field component parallel to the magnetic field. Spin precession about the resulting radial magnetic field component mimics an EDM signal. It can be eliminated by injecting the beam clockwise and counter-clockwise. The sign of the signals are opposite, since $\beta$ changes sign, but $E$ (and thus $E_{z}$ ) remains the same.

A limitation of this method is the maximal strength of the radial electric field. Therefore, this technique is only suited for particles with small anomalous magnetic moments. Detailed studies for the muon and deuterons show that limits of $\left|d_{\mu}\right|<$ $10^{-24} \mathrm{e} \cdot \mathrm{cm}$ and $\left|d_{\mathcal{D}}\right|<10^{-27} \mathrm{e} \cdot \mathrm{cm}$ are reachable $[11,12]$.

\subsection{Spin resonance}

The limitation imposed by the radial electric field can be avoided by yet another technique, the so-called resonance method [13]. For a time dependent velocity $\beta_{0}+\delta \beta(t)$ generated by an oscillating longitudinal electric field $E_{R F}(t)$ and a constant magnetic field, the spin evolution follows from

$$
\begin{aligned}
\frac{d \vec{S}}{d t} & =\vec{S} \times \frac{e}{m}\left[\left\{a B+\frac{\eta}{2} \vec{\beta}_{0} \times \vec{B}\right\}+\frac{\eta}{2}\left\{\delta \vec{\beta}(t) \times \vec{B}+\frac{1}{\gamma} \vec{E}_{R F}(t)\right\}\right] \\
& \equiv \vec{S} \times[\vec{\Omega}+\delta \vec{\Omega}(t)]
\end{aligned}
$$

The second term can be treated as a perturbation, because $\Omega \gg d \Omega(\propto \eta)$. The first term yields spin precession about $\vec{\Omega}$, without affecting the polarization parallel to it. For $\delta \beta(t)=\delta \beta \cos (\omega t+\psi)$, the parallel polarization component is given by

$$
\begin{aligned}
d P_{\|} / d t \simeq\left(\vec{P}_{\perp} \times \delta \vec{\Omega}\right)_{\|} & =P_{\circ} \cos (\Omega t+\phi) \times \delta \Omega \cos (\omega t+\psi) \\
& \left.=\frac{1}{4} \frac{e}{m} P_{\circ} \eta \delta \beta B \cos (\Delta \omega t+\Delta \phi)\right) .
\end{aligned}
$$


In the last step, fast oscillating terms $(\propto \cos ((\Omega+\omega) t))$ were omitted. Further, $\Delta \omega \equiv \Omega-\omega$ and $\Delta \phi \equiv \phi-\psi$. For $\Delta \omega=0$ the vertical polarization will grow continuously at a rate proportional to the EDM. Maximum sensitivity is obtained for $\Delta \phi=0$ or $\pi$.

The statistical sensitivity of this method $(\alpha \delta \beta B / 2)$ is similar to that for the 'frozen spin' method $(\alpha \beta B)$, because there is no limit on the magnetic field strength.

Systematic errors could appear if some field perturbation oscillates in resonance with the spin and the velocity, e.g. in a section of the ring that exhibits dispersion. An independent measure of how these perturbations produce a fake EDM signal is obtained by tuning the vertical beam oscillation (betatron) frequency. In normal operation, this frequency is far from resonance, and has little sensitivity. Close to resonance, the sensitivity is several orders of magnitude larger. Simultaneous storage of bunches with different vertical betatron frequencies (possible with a fast switching quadrupole), allows to reduce this systematic sensitivity to less than $10^{-29} \mathrm{e} \cdot \mathrm{cm}$. Another powerful tool to control systematic uncertainties is the possibility to simultaneously store bunches each with a different $\Delta \phi$ and thus different EDM sensitivity.

This method is in principle suitable for all particles. However, a practical experiment consists of a storage ring with a single accelerating cavity, limiting the method to particles for which the number of spin revolutions per turn is $a \gamma \simeq 0$, $1, \cdots$. Further (weak) limitations are imposed by the frequency and voltage of the cavity. The method was initially aimed at the search for a deuteron EDM $(a=$ $-0.143)$. Presently, it is believed that also other light ion EDMs might be within reach, such as the proton $(a=1.79)$ and ${ }^{3} \mathrm{He}(a=-4.19)$. For all these nuclei, polarized sources are available and polarimetry based on the spin dependence in nuclear scattering yields acceptable efficiency.

\section{Conclusion and outlook}

Three methods were described to directly measure permanent electric dipole moments of charged particles. All methods make use of the strong motional electric field relativistic particles experience while moving in a magnetic storage ring. In the particle frame, the coupling of the magnetic and electric dipole moments to the magnetic and electric fields cause spin precession.

To date, the only direct measurement of a charged particle EDM that reaches reasonable precision was made for the muon $\left(\left|d_{\mu}\right|<\mathcal{O}\left(10^{-19}\right) \mathrm{e} \cdot \mathrm{cm}\right)$. It was obtained in an experiment aimed to measure the anomalous magnetic moment. The EDM was extracted from the tilt in the spin precession plane. The sensitivity of this method is limited by systematic uncertainties.

A first step towards increased sensitivity can be made by 'freezing' the precession caused by the anomalous magnetic moment, using a radially oriented electric field. It is expected that in a dedicated experiment, the EDM measurement on the muon can be improved by some five orders of magnitude. The method of frozen spin is applicable for all particles with a sufficiently small anomalous magnetic moment, the magnitude of which determines the strength of the radial electric field. The largest systematic uncertainty for this method originates from a vertical electric field component. For the deuteron (one of the candidate particles), this systematic error is estimated to be of the order of $10^{-27} \mathrm{e} \cdot \mathrm{cm}$. 
The second step is to remove this large systematic error. By setting up a resonance between the spin and the velocity modulation, the radial electric field is no longer needed. Because of the residual spin precession, static perturbations decouple from the EDM signal. EDM limits of the order of $10^{-29} \mathrm{e} \cdot \mathrm{cm}$ seem reachable with this method. Already at a sensitivity of $10^{-24} \mathrm{e} \cdot \mathrm{cm}$, which seems readily achievable, several speculative new physics models can be tested [14].

Acknowledgements The author is financed by grant 680-47-203 of the Innovational Research Incentives Scheme of the Netherlands Organization for Scientific Research (NWO).

\section{References}

1. Jungmann, K.: Fundamental interactions. In: these proceedings.

2. Khriplovich, I.B., Lamoreaux, S.K.: CP Violation without Strangeness. Springer, Berlin Heidelberg New York (1997)

3. Sandars, P.G.H.: Electric dipole moments of charged particles. Contemp. Phys. 42, 97 (2001)

4. Liu, C.P., Timmermans, R.G.E.: P- and T-odd two-nucleon interaction and the deuteron electric dipole moment. Phys. Rev., C 70, 055501 (2004)

5. Lebedev, O., Olive, K.A., Pospelov, M., Ritz, A.: Probing CP violation with the deuteron electric dipole moment. Phys. Rev., D 70, 016003 (2004)

6. Purcell, E.M., Ramsey, N.F.: On the possibility of electric dipole moments for elementary particles and nuclei. Phys. Rev. 78, 807 (1950)

7. Schiff, L.I.: Measurability of nuclear electric dipole moments. Phys. Rev. 132, 2194 (1963)

8. Bailey, J., et al.: CERN Muon Storage Ring Collaboration: new limits on the electric dipole moment of positive and negative muons. J. Phys., G 4, 345 (1978)

9. McNabb, R.: Muon g-2 Collaboration: an improved limit on the electric dipole moment of the muon. arXiv:hep-ex/0407008 (2004)

10. Farley, F.J.M., et al.: A new method of measuring electric dipole moments in storage rings. Phys. Rev. Lett. 93, 052001 (2004)

11. Silenko, A., et al.: J-PARC letter of intent L22: search for a permanent Muon electric dipole moment at the $10^{-24} \mathrm{e} \cdot \mathrm{cm}$ level. Available at http://www.bnl.gov/edm/papers/jparc_loi_030109. pdf (2003)

12. Aoki, M., et al.: BNL proposal P970: search for a permanent deuteron electric dipole moment at the $10^{-27} \mathrm{e} \cdot \mathrm{cm}$ level. Available at http://www.bnl.gov/edm/deuteron_proposal_040816.pdf (2004)

13. Orlov, Y.F., Morse, W.M., Semertzidis, Y.K.: Resonance method of electric-dipole-moment measurements in storage rings. Phys. Rev. Lett. 96, 214802 (2006)

14. Pospelov, M., Ritz, A.: Electric dipole moments as probes of new physics. Ann. Phys. 318, 119 (2005) 